OPEN ACCESS

Edited by:

Shuofeng Yuan,

The University of Hong Kong,

Hong Kong, SAR China

Reviewed by:

Jun Wang,

University of Arizona, United States

Xiaopan Gao,

Institute of Pathogen Biology (CAMS),

China

*Correspondence:

Md. Golzar Hossain

mghossain@bau.edu.bd

Md. Sayeedul Islam

islam@bio.sci.osaka-u.ac.jp

Specialty section:

This article was submitted to

Virology,

a section of the journal

Frontiers in Microbiology

Received: 02 July 2021

Accepted: 18 August 2021 Published: 17 September 2021

Citation:

Hossain MG, Akter S, Dhole P.

Saha S, Kazi T, Majbauddin A and Islam MS (2021) Analysis of the

Genetic Diversity Associated With

the Drug Resistance

and Pathogenicity of Influenza A Virus

Isolated in Bangladesh From 2002

to 2019. Front. Microbiol. 12:735305.

doi: 10.3389/fmicb.2021.735305

\section{Analysis of the Genetic Diversity Associated With the Drug Resistance and Pathogenicity of Influenza A Virus Isolated in Bangladesh From 2002 to 2019}

\author{
Md. Golzar Hossain ${ }^{*}$, Sharmin Akter ${ }^{2}$, Priya Dhole ${ }^{3}$, Sukumar Saha ${ }^{1}$, \\ Taheruzzaman Kazi ${ }^{4}$, Abir Majbauddin ${ }^{4}$ and Md. Sayeedul Islam ${ }^{5 *}$
}

${ }^{1}$ Department of Microbiology and Hygiene, Bangladesh Agricultural University, Mymensingh, Bangladesh, ${ }^{2}$ Department of Physiology, Bangladesh Agricultural University, Mymensingh, Bangladesh, ${ }^{3}$ Department of Biology, The Pennsylvania State University, Pennsylvania, PA, United States, ${ }^{4}$ Department of Regenerative Dermatology, Graduate School of Medicine, Osaka University, Osaka, Japan, ${ }^{5}$ Department of Biological Sciences, Graduate School of Science, Osaka University, Osaka, Japan

The subtype prevalence, drug resistance- and pathogenicity-associated mutations, and the distribution of the influenza A virus (IAV) isolates identified in Bangladesh from 2002 to 2019 were analyzed using bioinformatic tools. A total of 30 IAV subtypes have been identified in humans (4), avian species (29), and environment (5) in Bangladesh. The predominant subtypes in human and avian species are H1N1/H3N2 and H5N1/H9N2, respectively. However, the subtypes H5N1/H9N2 infecting humans and H3N2/H1N1 infecting avian species have also been identified. Among the avian species, the maximum number of subtypes (27) have been identified in ducks. A 3.56\% of the isolates showed neuraminidase inhibitor (NAl) resistance with a prevalence of 8.50 , 1.33, and $2.67 \%$ in avian species, humans, and the environment, respectively, the following mutations were detected: V116A, I117V, D198N, I223R, S247N, H275Y, and N295S. Prevalence of adamantane-resistant IAVs was 100, 50, and 30.54\% in humans, the environment, and avian species, respectively, the subtypes H3N2, H1N1, H9N2, and $\mathrm{H} 5 \mathrm{~N} 2$ were highly prevalent, with the subtype H5N1 showing a comparatively lower prevalence. Important PB2 mutations such D9N, K526R, A588V, A588I, G590S, Q591R, E627K, K702R, and S714R were identified. A wide range of IAV subtypes have been identified in Bangladesh with a diversified genetic variation in the NA, M2, and PB2 proteins providing drug resistance and enhanced pathogenicity. This study provides a detailed analysis of the subtypes, and the host range of the IAV isolates and the genetic variations related to their proteins, which may aid in the prevention, treatment, and control of IAV infections in Bangladesh, and would serve as a basis for future investigations.

Keywords: influenza A virus, subtypes, host, mutations, drug resistance, pathogenicity, Bangladesh 


\section{INTRODUCTION}

Influenza caused by influenza viruses (types A, B, C, and D) is a contagious respiratory infection distributed worldwide. Influenza has a wide host range depending on the strains of the influenza $\mathrm{A}$ virus (IAV). Based on the circulating strains of the influenza virus, a myriad range of hosts have been described. Many avian and mammalian species such as chicken, duck, quail, and crow, and human, swine, horse, and cat, respectively, might be infected with IAV (Taubenberger and Kash, 2010; Hussain et al., 2017). Every year, millions of people are infected with influenza worldwide, with increasing economic losses due to diagnosis, treatment, and vaccine development costs (Molinari et al., 2007; Mao et al., 2012; Palekar et al., 2019). Likewise, some strains of the IAV with a high morbidity and mortality rate severely impact the poultry industries (Thomas and Noppenberger, 2007; Yoo et al., 2018).

IAV is an enveloped virus of around $100-\mathrm{nm}$ diameter. It belongs to the Alphainfluenzavirus genus and Orthomyxoviridae family. It is a segmented RNA virus, which is negative-sense, single-stranded, and contains eight RNA segments (Eisfeld et al., 2015). The entire genome is around 13,588 bases encoding at least 10 viral proteins (Eisfeld et al., 2015). The major proteins encoded by the viral genomes are hemagglutinin (HA), neuraminidase (NA), nucleoprotein (NP), matrix proteins (M1 and M2), polymerase proteins (PB1, PB2, and PA), and nonstructural proteins (NS1 and NEP) (McCauley and Mahy, 1983).

The HA and NA proteins are present on the outermost surface of the virion and play crucial roles in the attachment of the virus to the host cells to initiate the infection cycle, leading to the subsequent release of the viral progeny (Suzuki, 2005; Cohen et al., 2013). The currently available anti-influenza drugs primarily target the NA protein (Wilson and von Itzstein, 2003). The IAV can be classified into different subtypes based on the antigenic properties of the HA and NA proteins. To date, $16 \mathrm{HA}$ and 9 NA subtypes have been reported based on their amino acid variations (Lynch and Walsh, 2007; Proença-Módena et al., 2007). The HA protein determines the host specificity of IAV. The HA proteins of the avian and human influenza viruses bind with two to three sialic acid and two to three sialic acid receptors, respectively, whereas the swine influenza binds both (Trebbien et al., 2011; Byrd-Leotis et al., 2017). However, many of IAV subtypes, H5N1, H7N9, and H9N2, can infect both birds and humans (Mostafa et al., 2018). On the other hand, the M2 protein activated during the IAV entry into the host cells releases the ribonucleoprotein into the cytoplasm to initiate the viral replication (Jalily et al., 2020). The drug adamantane blocks the viral replication during the uncoating step by inhibiting the IAV M2 ion transport (Takeda et al., 2002; Jalily et al., 2020). However, mutations in the NA and M2 proteins might affect the drug susceptibility, and resistant IAV strains have been reported (Hussain et al., 2017). Drug-resistant and mutated highly pathogenic IAVs are distributed worldwide (Wen et al., 2018; Moasser et al., 2019).

Surveillance, vaccines, and antiviral drugs are essential to control and eradicate IAV from a region (Hussain et al., 2017). The rapid spread of drug-resistant IAVs interrupts the management and prevention strategies (Ormond et al., 2017).
Hence, researchers have been investigating and designing various next-generation antivirals that are effective against drug-resistant IAVs (Shin and Seong, 2019; Musharrafieh et al., 2020; Toots and Plemper, 2020; Yin et al., 2021). In addition, current influenza vaccines provide only partial protection and may fail to protect against the mutated and novel variants of IAVs (Vanderlinden and Naesens, 2014; Paules and Fauci, 2019). Therefore, the surveillance and analysis of the IAV mutations associated with drug resistance are important for the prevention and treatment of IAV infection.

Among the three subunits of IAV polymerases (PB1, PB2, and PA), PB2 is an important virulence determinant (Graef et al., 2010). PB2 initiates the genome replication of IAV by modulating the ribonucleoprotein complex and regulating the host immune system and antiviral signaling pathways (Carr et al., 2006; Graef et al., 2010). Many clinical and experimental studies have shown that mutations in PB2 affect the activity of this polymerase and the pathogenicity of IAV (Tian et al., 2012; de Jong et al., 2013; Song et al., 2014; Wen et al., 2018). PB2 mutations are also responsible for the interspecies transmission of IAV (Yamada et al., 2010; Wen et al., 2018; Wang et al., 2019). Therefore, the mutational analysis of $\mathrm{PB} 2$ in terms of the pathogenicity and interspecies transmission of IAV will be very helpful in preventing and controlling IAV infections.

Human and avian IAV infections were reported in Bangladesh in 2002 and 2006, respectively (Chi et al., 2005; Rimi et al., 2019). On the other hand, highly pathogenic avian influenza (HPAI) H5N1 was reported in poultry in 2007 for the first time and posed a serious threat to public health (Rimi et al., 2019). Moreover, HPAI H5N1 and low pathogenic avian influenza (LPAI) H9N2 caused significant damage to the poultry industry in Bangladesh (Turner et al., 2017; Kim, 2018; Parvin et al., 2018). In addition, the genetic reassortment of HPAI and the introduction of the new clades of IAV are now very common in Bangladesh (Parvin et al., 2014; Marinova-Petkova et al., 2016; Nooruzzaman et al., 2019). However, to our knowledge, the overall prevalence of the different IAV subtypes and their host range in Bangladesh have not yet been reported. The analysis of the genetic mutations associated with drug resistance and pathogenicity is necessary for the development of vaccines and treatment strategies to eradicate IAV from Bangladesh. Therefore, this study analyzed the overall prevalence of the IAV subtypes in Bangladesh from 2002 to 2019 along with the determination of their specific host distribution. Moreover, mutational analyses were performed to determine the prevalence of the drug resistance-associated mutations in the NA and M2 proteins and the pathogenicity-associated mutations in the $\mathrm{PB} 2$ protein of IAV.

\section{MATERIALS AND METHODS}

\section{Collection and Processing of Influenza A Virus Sequences}

The genome sequences of the IAV isolates from Bangladesh were retrieved from GISAID. ${ }^{1}$ The data about IAV isolates

${ }^{1}$ https://www.gisaid.org/ 
deposited between 2002 and 2019 in GISAID was acquired. The sequences were either full or partial. The IAV isolates from Bangladesh were cross-checked with the NCBI influenza virus database (Bao et al., 2007) and Influenza Research Database. ${ }^{2}$ The retrieved sequences were downloaded, processed, and analyzed using several bioinformatic tools, such as CLC Sequence viewer, ${ }^{3}$ NCBI influenza virus database (Bao et al., 2007), and Influenza Research Database (see text footnote 2). The CLC Sequence viewer is a basic bioinformatics tool used to view, create, and edit alignments, and analyze the genomic sequences. The Influenza Virus Sequence Annotation Tool of the NCBI influenza virus database is an online application that can predict influenza protein sequences coded by the nucleotide sequences with specific drug resistance- and virulence-associated mutations. The Influenza Research Database contains surveillance data of the non-human and avian species and the clinical data of humans with the phenotypic, genomic, and proteomic data of the isolated virus strain. This database is used to analyze and visualize various features such as alignment, point mutations in specific proteins, and drug resistance (see text footnote 2 ).

\section{Analysis of the Prevalence of the Influenza A Virus Subtypes}

A total of 2,005, either full or partial genome sequences of IAV isolates, were used to determine subtype prevalence. According to the GenBank and NCBI Influenza Virus Database, a few sequences were designated as mixed by the sequence submitter. On the other hand, the subtype of some sequences could not be determined by the sequence submitter. Therefore, these mixed or undetermined subtypes were excluded from the analysis. The sequences were screened either using the NCBI influenza virus database or Microsoft Excel for the specific subtypes and their host distribution, and the prevalence was analyzed accordingly.

\section{Analysis of Drug Resistance-Associated Mutations in Neuraminidase and M2 Proteins}

A total of 1,828 amino acid sequences of the viral NA protein of the IAV isolates were analyzed for mutations associated with neuraminidase inhibitor (NAI) resistance. Among these, 1,200, 553, and 75 sequences were from human-, avian-, and environment-derived IAVs, respectively. The mutations were determined using the "Antiviral Resistance Risk Assessment" tool of the Influenza Research Database (Zhang et al., 2016). Based on the previously submitted data, this tool determines the amino acid mutations in the sequences associated with the alteration of susceptibility to antiviral drugs. A total of 1,761 amino acid sequences of M2 protein were retrieved (human: 1,220, avian: 465, and environment: 76), and adamantane resistanceassociated mutations were analyzed using the "Identify Point Mutations in Proteins" tool of the Influenza Research Database (Bao et al., 2007; Dong et al., 2015). This tool had three parameters set as default: type of protein to scan for the specified

${ }^{2}$ https://www.fludb.org/

${ }^{3}$ http://www.clcbio.com mutation (s), specific mutation coordinate, and specific subtype (s). Within these parameters, the subtype, protein, and mutation name with their amino acid positions were changed every time based on the purpose of the analysis.

\section{Analysis of the PB2 Amino Acid Mutations Associated With Influenza A Virus Virulence}

In total, 1,568 amino acid sequences of the PB2 protein were analyzed for the mutations associated with IAV virulence using the "Identify Point Mutations in Proteins" tool of the Influenza Research Database (Bao et al., 2007; Kim et al., 2010; Wen et al., 2018). Of these, 1,050, 456, and 62 sequences were from human-, avian-, and environment-derived IAVs.

\section{RESULTS}

\section{Prevalence of Influenza A Virus Subtypes in the Human, Environmental, and Avian Species}

The human IAVs were first reported in Bangladesh in 2002, whereas avian influenza was reported in 2006, according to the GenBank information (Chi et al., 2005; Bao et al., 2007; Parvin et al., 2014). Furthermore, a highly pathogenic IAV (H5N1) was reported in 2007 (Rimi et al., 2019). However, during the last two decades, many IAV subtypes have been reported sporadically in Bangladesh. Therefore, the overall prevalence of the identified subtypes in Bangladesh, from 2002 to 2019 from a total of 2,005 IAV isolates, was analyzed (human: 1,311, avian: 618 , and environment: 76). To date, 30 subtypes of IAV have been identified in Bangladesh among which only 4 infect humans, 5 were from non-human and non-avian environmental sources, and 29 subtypes infect avian species (Figure 1A). Among the avian IAV subtypes, the subtype H5N1 is highly prevalent $(56.80 \%)$ followed by H9N2 $(26.70 \%)$ (Figure 1B). The prevalence of the subtypes H3N2 (52.48\%) and H1N1 (47.22\%) in humans was higher though sporadic infections were found with H5N1 (0.23\%) and H9N2 (0.08\%) (Figure 1C). However, in the environment, the prevalence of H9N2 was higher $(60.53 \%)$ compared with that of H5N1 (34.21\%), H7N9 (2.63\%), H5N3 (1.32\%), and $\mathrm{H} 11 \mathrm{~N} 3$ (1.32\%) (Figure 1D). In summary, among the 30 IAV subtypes circulating in Bangladesh, H5N1 and H9N2 predominantly infect avian hosts or are primarily isolated from the environment, whereas $\mathrm{H} 3 \mathrm{~N} 2$ and $\mathrm{H} 1 \mathrm{~N} 1$ infect humans. The subtypes $\mathrm{H} 5 \mathrm{~N} 1$ and $\mathrm{H} 9 \mathrm{~N} 2$ were also sporadically identified in humans though they are primarily found in the environment and avian species.

\section{Distribution of the Influenza A Virus Subtypes in Various Species of Birds}

As 29 out of the reported 30 subtypes of IAV (618 isolates) have been found in avian species, the distribution of the specific subtypes in different avian hosts was analyzed. These 29 subtypes can infect 10 different avian species (Figure 2). The maximum number of subtypes (27) were isolated from ducks followed by 
A

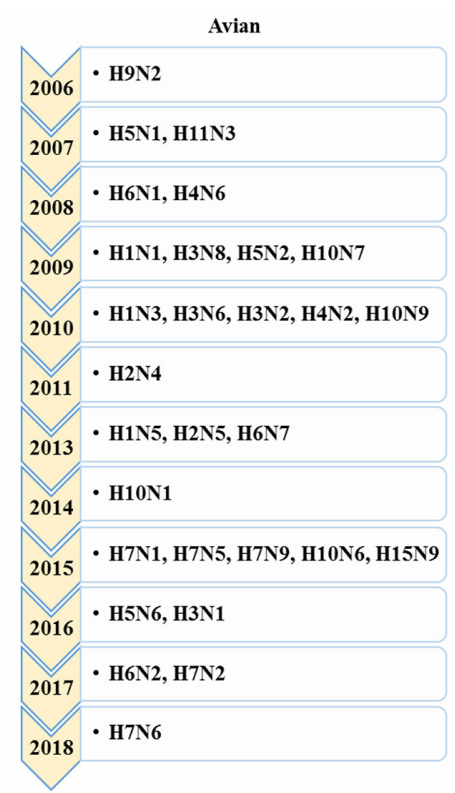

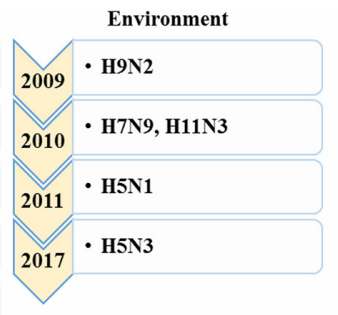

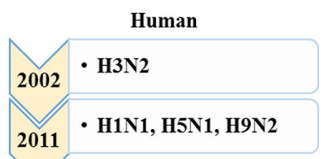

B

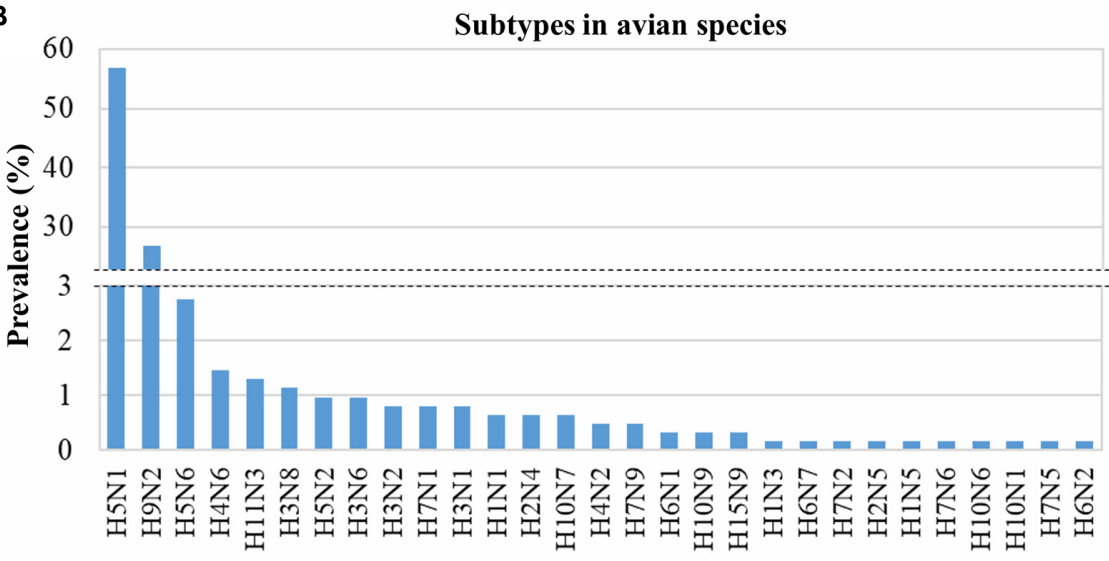

C
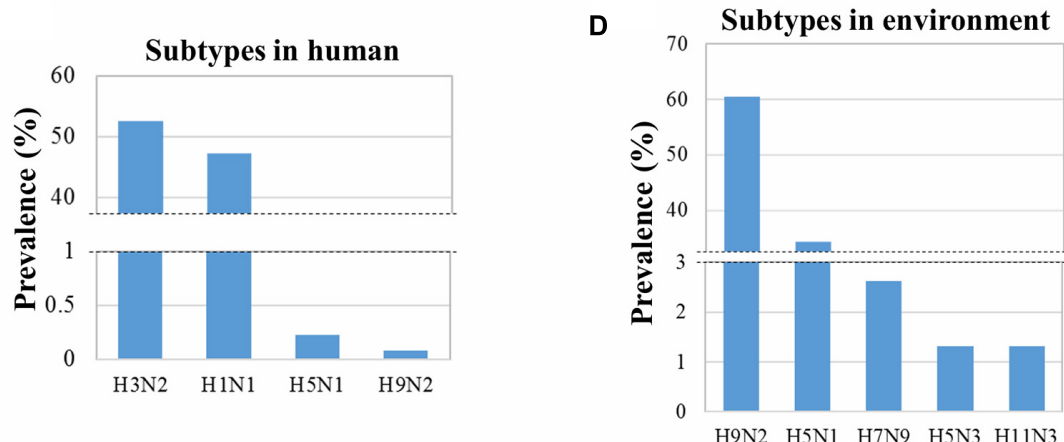

FIGURE 1 | Emergence and prevalence of influenza A virus (IAV) subtypes in humans, environment, and avian species. Data regarding 2,005 IAV isolates (human: 1,311, avian: 618, and environment: 76) collected in Bangladesh from 2002 to 2019 were retrieved from GISAID, and the prevalence of the different subtypes was determined. (A) Year of first reporting of the emergence of subtypes from 2002 to 2019. Prevalence of IAV subtypes in the avian hosts (B), humans (C), and environment (D).

chickens (5) and two each were found in quails, geese, and waterfowls; the remaining five avian species were each found to host only one subtype (Figure 2). Moreover, H5N1 was found to be distributed among the highest number of avian species (eight), followed by H9N2 (four species), H5N6 (three species), and H5N2 and H10N7 (two species each) (Figure 2). Next, the 

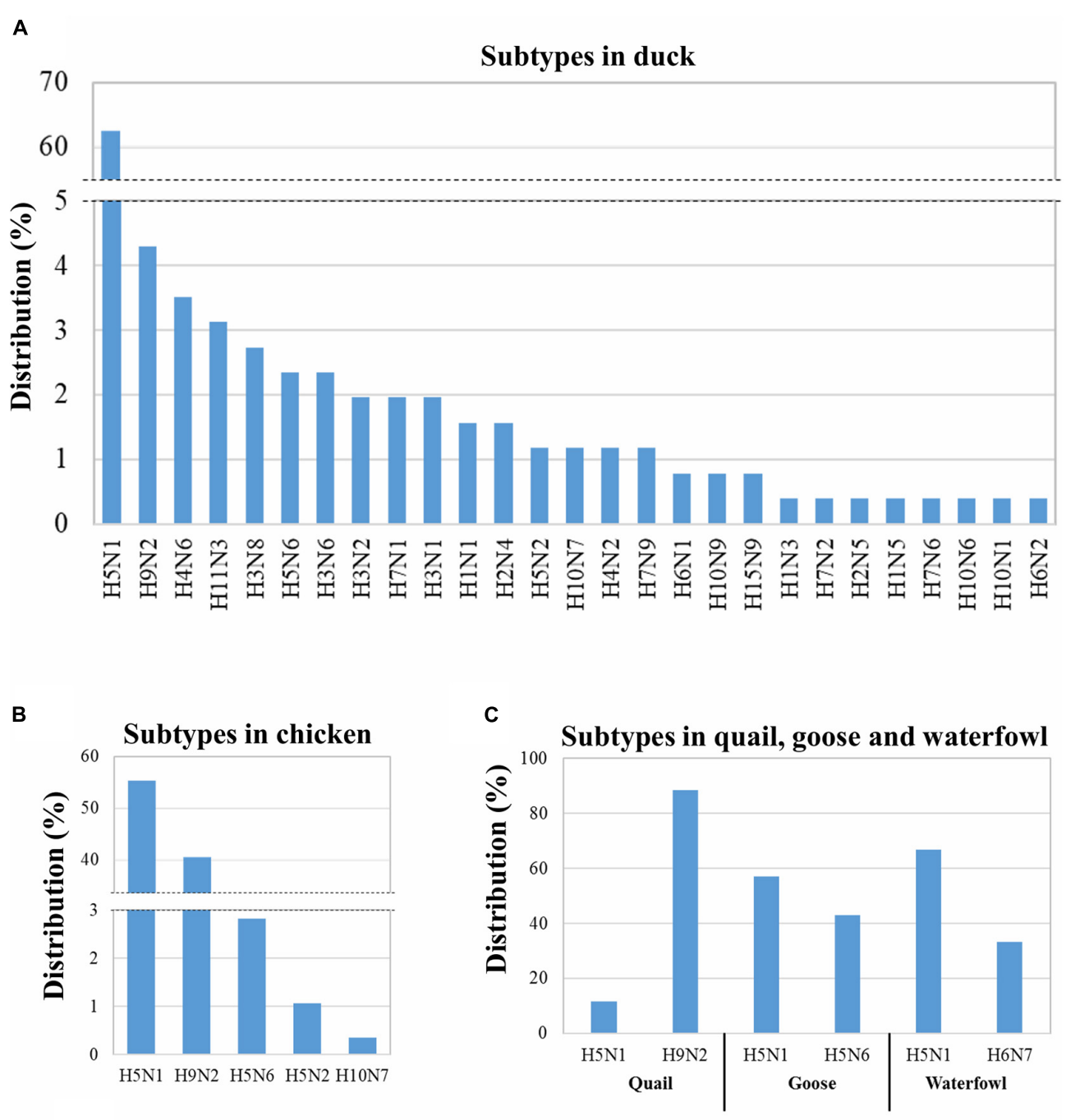

D

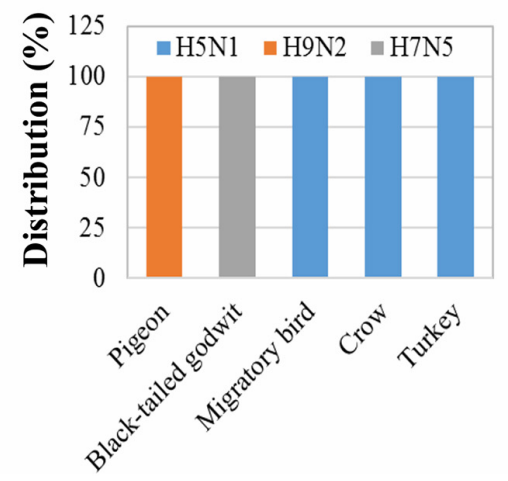

FIGURE 2 | Distribution of the IAV subtypes in various avian species in Bangladesh. Distribution was analyzed using 618 IAV isolates from 2006 to 2019 retrieved from GISAID. Distribution of 256 IAV duck isolates into subtypes (A). Distribution of 284 chicken, 43 quail, 7 goose, and 3 waterfowl IAV isolates among subtypes (B,C). Distribution of mentioned avian IAV isolates in different subtypes (D).

prevalence of the different subtypes in the specific avian hosts was analyzed. From the total of 27 IAV subtypes identified in ducks, $62.5 \%$ were H5N1 (Figure 2A). In addition, among the five subtypes circulating in chicken, H5N1 (55\%) and H9N2 (40.49\%) were found to be highly prevalent (Figure 2B). However, at least two subtypes were identified in quails, geese, and waterfowls 
(Figure 2C). The other five avian species showed the prevalence of only one subtype of IAV (Figure 2D). In conclusion, though 29 IAV subtypes were identified in the avian hosts in Bangladesh, the subtypes $\mathrm{H} 5 \mathrm{~N} 1$ and $\mathrm{H} 9 \mathrm{~N} 2$ were found to be the most predominant. A large number of subtypes (27) were determined to be circulating in ducks.

\section{Analysis of Neuraminidase Inhibitor Resistance-Associated Mutations}

Some IAV isolates show resistance against NAIs due to mutations in their neuraminidase (NA) proteins (Abed et al., 2006; Zürcher et al., 2006; Orozovic et al., 2011; WHO, 2018; Moasser et al., 2019). Based on the analysis of 1,828 isolates/sequences of IAV NA proteins, we found 65 IAV isolates showing resistance to oseltamivir and two showing resistance to oseltamivir and zanamivir. The overall prevalence of NAI-resistant IAV isolates was $3.56 \%(65 / 1,828)$. The prevalence in humans, birds, and the environment was $1.33 \%(16 / 1220), 8.50 \%(47 / 553)$, and $2.67 \%(2 / 75)$, respectively (Figure 3A). The distribution of the NAI-resistant human and environmental isolates was limited to $\mathrm{H} 1 \mathrm{~N} 1$ and H7N9. The avian IAV isolates resistant to NAI were distributed among a wide range of subtypes (Figure 3C). Among 47 NAI-resistant avian IAV isolates, $78.72 \%(37 / 47)$ were found in ducks, $12.76 \%$ in chickens, $6.38 \%$ in geese, and $2.12 \%$ in waterfowls (Figure 3B). The corresponding mutations were V116A, I117V, D198N, D199M, I223R, S247N, H275Y, and N295S, which were previously shown to occur naturally in the infected hosts (Table 1).

\section{Analysis of the Mutations in the M2 Protein Associated With Adamantane Resistance}

Several mutations, such as L26F, V27A, A30T, A30V, S31N, and $\mathrm{G} 34 \mathrm{E}$ in the M2 protein, have been reported to be associated with the adamantane resistance of IAVs isolated from infected hosts (Bao et al., 2007; Dong et al., 2015). The prevalence of adamantane-resistant isolates was $78.88 \%$ (1389/1761); these isolates were limited to only five subtypes: H9N2, H5N1, H3N2, H1N1, and H5N2 (Figure 4B). Almost 100\% $(1,209 / 1,220)$ of the human IAV isolates from Bangladesh were found to be resistant to adamantanes, followed by the environment-derived IAVs (50\%; 38/76), and the avian host-derived IAVs $(30.54 \%$; 142/465) (Figure 4A). Moreover, almost $100 \%$ of the H3N2, $\mathrm{H} 1 \mathrm{~N} 1$, and H9N2 subtypes were adamantane-resistant, whereas the least number of the $\mathrm{H} 5 \mathrm{~N} 1$ isolates (6.67\%) showed resistance to adamantanes (Figure 4B). Besides, in the case of avian isolates, the adamantane-resistant IAVs were found only in chickens, ducks, quails, and pigeons (Figure 4C).

\section{Mutation in the PB2 Protein Associated With Virulence of Influenza A Viruses}

Mutations in the protein PB2 increase the viral polymerase activity and pathogenicity of the IAV (Tian et al., 2012; de Jong et al., 2013; Song et al., 2014; Wen et al., 2018). Our analysis showed that almost $100 \%$ of the human IAV isolates contained PB2 mutations. These mutations are required for

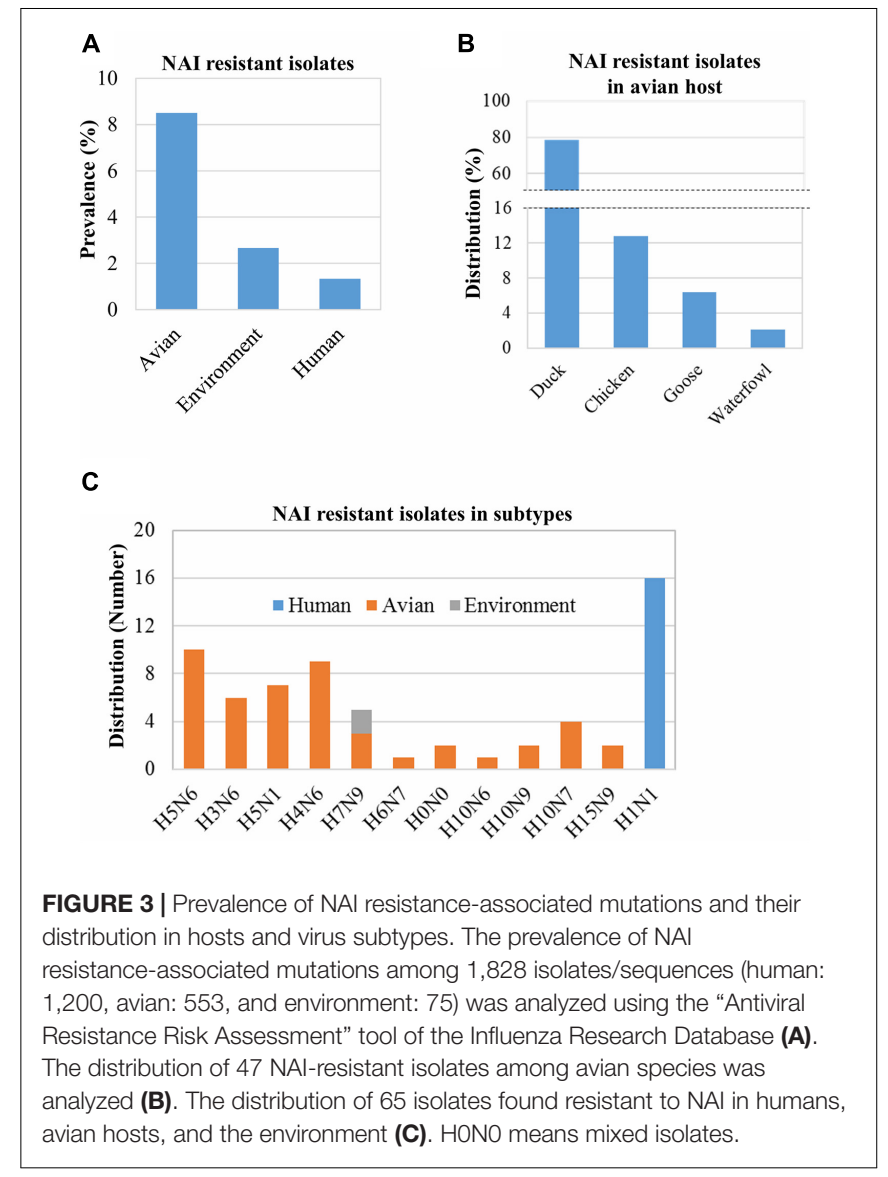

TABLE 1 | Mutations associated with the drug resistance and pathogenicity of the influenza A virus (IAV) isolates found in this study.

\begin{tabular}{|c|c|c|}
\hline Proteins & Mutations & Properties \\
\hline \multirow[t]{8}{*}{ NA } & V116A & Resistance to oseltamivir or zanamivir \\
\hline & $1117 \mathrm{~V}$ & \\
\hline & D198N & \\
\hline & D199N & \\
\hline & I223R & \\
\hline & S247N & \\
\hline & $\mathrm{H} 275 \mathrm{Y}$ & \\
\hline & N295S & \\
\hline \multirow[t]{3}{*}{ M2 } & L26F & Resistance to adamantanes \\
\hline & V27A & \\
\hline & S31N & \\
\hline \multirow[t]{7}{*}{ PB2 } & D9N & Increased pathogenicity \\
\hline & K526R & \\
\hline & A588I/N & \\
\hline & G590S/Q591R & \\
\hline & E627K & \\
\hline & K702R & \\
\hline & S714R & \\
\hline
\end{tabular}

adaptation in mammalian hosts, and many of them might be associated with the increased pathogenicity. Nearly all of these mutations belonged to $\mathrm{H} 1 \mathrm{~N} 1$ (46.19\%) and $\mathrm{H} 3 \mathrm{~N} 2$ 


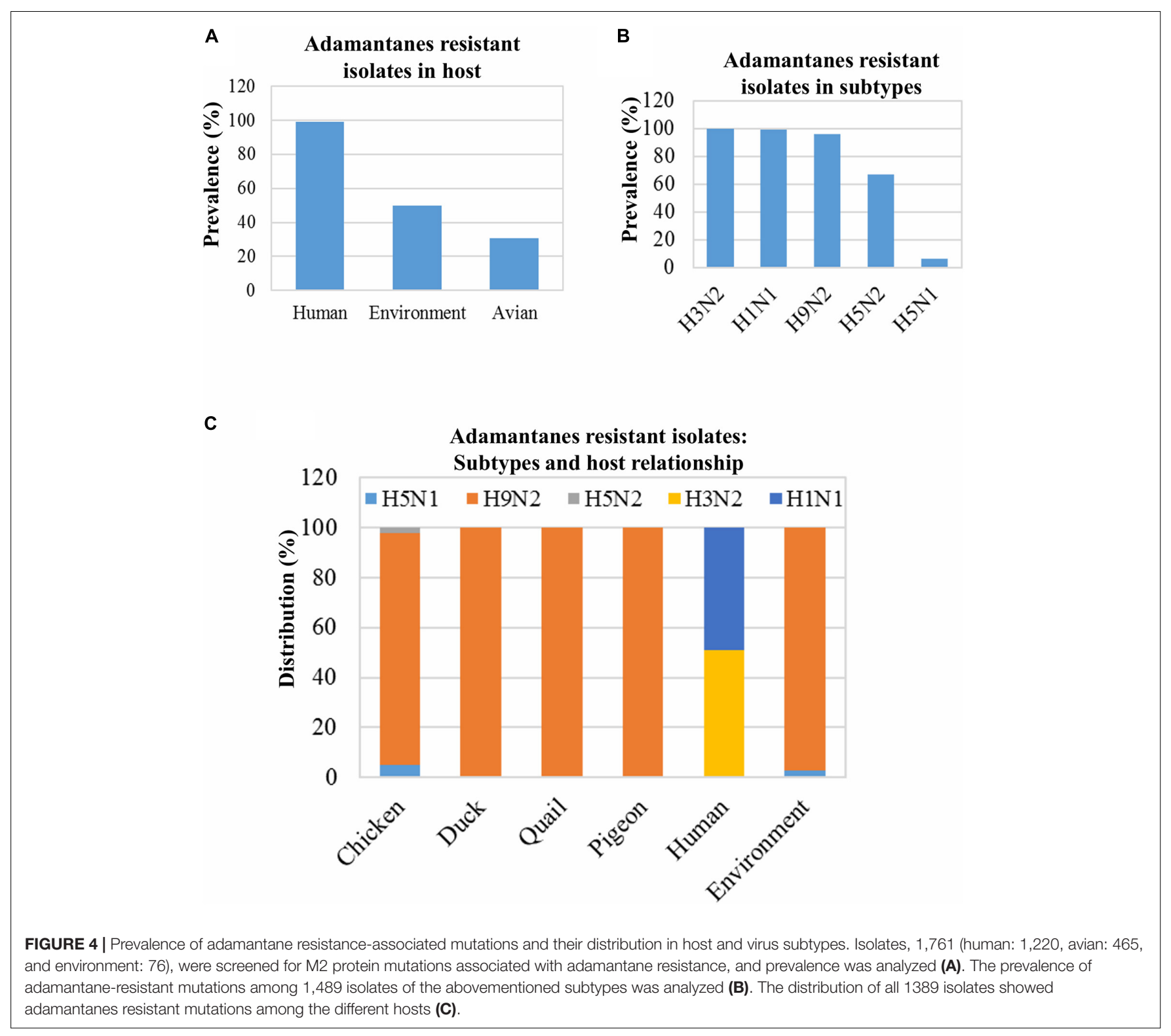

(53.52\%). The prevalence of mutated IAV isolates with increased pathogenicity was $48.46 \%(221 / 456)$ and $30.65 \%(19 / 62)$ in the avian host and environment, respectively. The mutations were found in the H5N1 (208/226), H9N2 (7/135), H5N2 $(2 / 3), \mathrm{H} 3 \mathrm{~N} 8$ (2/6), H7N1 (1/7), and H7N2 (1/1) isolates. Most of the PB2 mutations of avian IAVs were found in $\mathrm{H} 5 \mathrm{~N} 1$ $(208 / 226)$ and were primarily distributed in ducks (62\%) and chickens (28.96\%) (Figures 5A,B). All environmental isolates of H5N1 contain mutated PB2. Several mutations were identified in the sequences of the IAV isolates, namely, D9N, K526R, A588I/V, G590S/Q591R, E627K, K702R, and S714R. These mutations were previously reported to be associated with increased pathogenicity (Table 1; Tian et al., 2012; de Jong et al., 2013; Song et al., 2014; Wen et al., 2018). However, many isolates contained multiple mutations in PB2, especially isolates of human IAVs.

\section{DISCUSSION}

A total of $16 \mathrm{HA}$ and $9 \mathrm{NA}$ subtypes have been identified worldwide, and many of these have been reported as zoonotic (Proença-Módena et al., 2007; Mostafa et al., 2018). Several subtypes $(\mathrm{H} 1 \mathrm{~N} 1, \mathrm{H} 2 \mathrm{~N} 2$, and $\mathrm{H} 3 \mathrm{~N} 2)$ of IAV are pandemic in humans, but genetic reassortment of other zoonotic subtypes may increase the risk of infection in humans (Taubenberger and Kash, 2010; Chastagner et al., 2019). In the last few decades, mutations in the IAV genes have made the selection of appropriate antiviral drugs tremendously challenging. Therefore, proper IAV subtyping is essential for selecting the appropriate antiviral drug to cure the specific IAV infection and to identify new therapeutic targets, as several subtypes are resistant to the currently available antiviral drugs (Kaul et al., 2010; Parida et al., 2016). Moreover, IAV vaccine efficacy may vary depending on the 

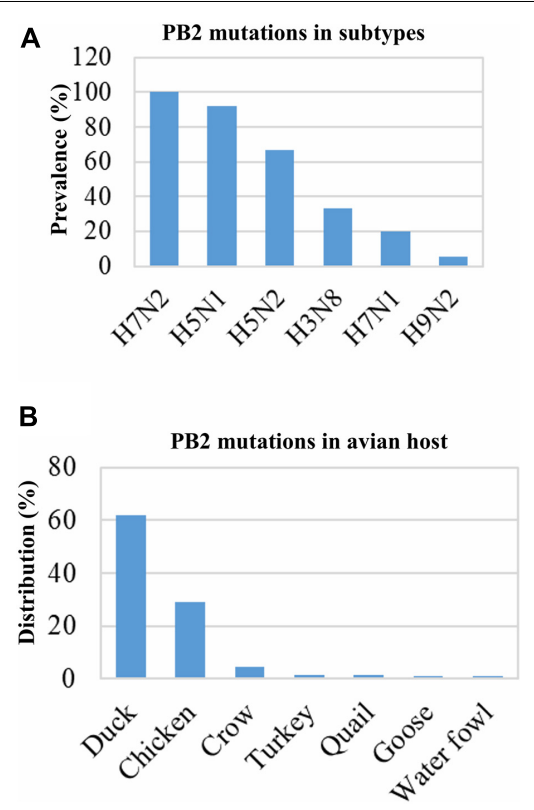

FIGURE 5 | Prevalence of PB2 protein mutations associated with increased pathogenicity and distribution in host and subtypes. Data of 1,568 isolates (human: 1,050, avian: 456, and environment: 62) were retrieved and analyzed. In avian hosts, 221 isolates showed PB2 mutations associated with increased pathogenicity, which were from 376 isolates of the abovementioned subtypes (A). Distribution of the 221 isolates among different avian species (B).

virus strain such as against a specific subtype, H3N2 (Belongia et al., 2016). IAV infects a wide range of hosts, and interspecies transmission is possible (Joseph et al., 2017). In this study, 30 subtypes of IAV were identified in Bangladesh from 2002 to 2019. Of these, 29 were found in avian species with a predominance of $\mathrm{H} 5 \mathrm{~N} 1$ and H9N2. Four human IAV isolates were reported, with 99.7\% belonging to H1N1 and H3N2 (Komadina et al., 2014). However, the interspecies transmission and genetic reassortment of IAVs occurred in Bangladesh and correlated with the global cases (Zhou et al., 2011; Lai et al., 2016; Briand et al., 2018). Interestingly both $\mathrm{H} 3 \mathrm{~N} 2$ and $\mathrm{H} 1 \mathrm{~N} 1$ were identified only in ducks, which were found to be infected with a total of 27 subtypes of IAVs in Bangladesh (Wilcox et al., 2011). Ducks might be reservoirs of various IAV subtypes, and coinfection with more than one subtype may be very common, and may facilitate the emergence of a more pathogenic new strain of IAV following genetic reassortment (Hinshaw et al., 1980; Sharp et al., 1997; Wilcox et al., 2011; Deng et al., 2013). Moreover, the emergence of $\mathrm{H} 3 \mathrm{~N} 2$ from ducks after genetic reassortment with other subtypes is alarming as novel virus strains infecting humans or other mammals may emerge (Zhou et al., 2011; El-Shesheny et al., 2018).

IAV-infected patients can be treated with two groups of anti-IAV drugs; adamantanes that block the viral M2 ion channel activity and NAIs, which inhibit the enzymatic activities of the viral NA protein (Hussain et al., 2017; Jalily et al., 2020; Musharrafieh et al., 2020). However, mutated adamantane-resistant IAV strains have been emerging since
1980 due to mutations in the M2 protein, and their prevalence is increasing (Heider et al., 1981; Dong et al., 2015). The IAV subtypes circulating in humans worldwide, especially the $\mathrm{H} 1 \mathrm{~N} 1$, and $\mathrm{H} 3 \mathrm{~N} 2$, show significant resistance to amantadine (Hussain et al., 2017). Few reports showed that the prevalence of adamantane-resistant IAVs exceeds 90\% in Asian countries (Nelson et al., 2009; Dong et al., 2015). Accordingly, more than 99\% of the human isolates in this analysis showed resistance to adamantanes, and they belong to the H1N1 and H3N2 subtypes. Moreover, more than $90 \%$ of H9N2 isolates from humans, avian hosts, and the environment also showed adamantane resistance. In addition, a small percentage of avian and environmental H5N1 isolates were also found to be resistant to adamantanes, consistent with previous findings (Cheung et al., 2006; Govorkova et al., 2013).

Though several NAIs are available in the market, oseltamivir and zanamivir have been used worldwide (Principi et al., 2019). However, mutations in the NA protein affecting the sensitivity of these drugs and IAV resistance have also emerged (Abed et al., 2006; Zürcher et al., 2006; Hussain et al., 2017; WHO, 2018; Moasser et al., 2019). During the study period of 2002 to 2019, $3.56 \%$ of the IAV isolates have been found to be resistant to oseltamivir. Interestingly the resistance rate is higher in avian species than in humans. All of the oseltamivir-resistant IAVs were of the H1N1 subtype, which is supported by the findings of a previous global analysis (Hussain et al., 2017). However, a wide range of IAV subtypes with NAI resistance were primarily found in ducks (Earhart et al., 2009; Järhult, 2012; Achenbach and Bowen, 2013).

Many mutations in the PB2 protein identified in human IAV isolates are present in avian species confirming the possibility of interspecies transmission (Yamada et al., 2010; Wen et al., 2018; Wang et al., 2019). PB2 mutations E627K and D701N are found in $\mathrm{H} 5 \mathrm{~N} 1$ among human isolates, whereas avian type PB2 exhibits the mutations 526K, 627E, and 701D (Chen et al., 2005; Mehle and Doudna, 2008). The PB2 K526R mutation is found in $\mathrm{H} 3 \mathrm{~N} 2$ among mammalian isolates, whereas the H1N1 subtype shows the mutation 590S/591R for replication in humans (Mehle and Doudna, 2009; Song et al., 2014). PB2 $\mathrm{K} 702 \mathrm{R}$ is also supposed to be a host marker as it is found in human $\mathrm{H} 5 \mathrm{~N} 1$ in Indonesia and has not been reported in avian species (Finkelstein et al., 2007). The mutational analysis of human IAV viral PB2 polymerase from 2002 to 2019 showed that all H1N1 subtypes contained G590S/Q591R mutations, whereas almost all of the H3N2 subtypes contained K526R, E627K, and K702R simultaneously. Interestingly, more than 90\% of the avian and $100 \%$ of the environmental H5N1 PB2 also contained the K526R, E627K, or K702R mutations, and these mutations were mostly distributed in ducks and chickens. This might be due to interspecies transmission and natural selection (Wang et al., 2019).

In summary, a total of 30 subtypes of the IAV were identified in Bangladesh. Among them, $\mathrm{H} 5 \mathrm{~N} 1$ and $\mathrm{H} 9 \mathrm{~N} 2$, are predominant in the avian host and environment, whereas H3N2 and H1N1 are prevalent in humans. The subtypes $\mathrm{H} 1 \mathrm{~N} 1, \mathrm{H} 5 \mathrm{~N} 1$, and $\mathrm{H} 9 \mathrm{~N} 2$ have been isolated from humans and avian species. A wide range of subtypes (27) has been found in ducks. All human IAV isolates 
are resistant to adamantanes. Though the prevalence is low, NAI-resistant IAVs have been circulating in Bangladesh. A high number of avian isolates contained PB2 mutations associated with increased pathogenicity in the human IAV.

\section{AUTHOR CONTRIBUTIONS}

MGH designed the study, extracted the sequences, data, and information from the online databases, analyzed the data, and wrote the manuscript. MGH, SA, PD, SS, TK, AM, and MSI edited, revised, and approved the final version of the

\section{REFERENCES}

Abed, Y., Baz, M., and Boivin, G. (2006). Impact of neuraminidase mutations conferring influenza resistance to neuraminidase inhibitors in the N1 and N2 genetic backgrounds. Antivir. Ther. 11, 971-976.

Achenbach, J. E., and Bowen, R. A. (2013). Effect of oseltamivir carboxylate consumption on emergence of drug-resistant $\mathrm{H} 5 \mathrm{~N} 2$ avian influenza virus in Mallard ducks. Antimicrob. Agents Chemother. 57, 2171-2181. doi: 10.1128/ AAC.02126- 12

Bao, Y., Bolotov, P., Dernovoy, D., Kiryutin, B., and Tatusova, T. (2007). FLAN: a web server for influenza virus genome annotation. Nucleic Acids Res. 35, W280-W284. doi: 10.1093/nar/gkm354

Belongia, E. A., Simpson, M. D., King, J. P., Sundaram, M. E., Kelley, N. S., Osterholm, M. T., et al. (2016). Variable influenza vaccine effectiveness by subtype: a systematic review and meta-analysis of test-negative design studies. Lancet Infect. Dis. 16, 942-951. doi: 10.1016/S1473-3099(16)00129-8

Briand, F.-X., Niqueux, E., Schmitz, A., Beven, V., Lucas, P., Allée, C., et al. (2018). Identification of a divergent avian influenza H3N2 virus from domestic ducks in France. Microbiol. Resour. Announc. 7, e00943-18. doi: 10.1128/MRA.0094318

Byrd-Leotis, L., Cummings, R. D., and Steinhauer, D. A. (2017). The Interplay between the host receptor and influenza virus hemagglutinin and neuraminidase. Int. J. Mol. Sci. 18:1541. doi: 10.3390/ijms18071541

Carr, S. M., Carnero, E., García-Sastre, A., Brownlee, G. G., and Fodor, E. (2006). Characterization of a mitochondrial-targeting signal in the PB2 protein of influenza viruses. Virology 344, 492-508. doi: 10.1016/j.virol.2005.08.041

Chastagner, A., Bonin, E., Fablet, C., Quéguiner, S., Hirchaud, E., Lucas, P., et al. (2019). Virus persistence in pig herds led to successive reassortment events between swine and human influenza A viruses, resulting in the emergence of a novel triple-reassortant swine influenza virus. Vet. Res. 50:77. doi: 10.1186/ s13567-019-0699-y

Chen, H., Smith, G. J. D., Zhang, S. Y., Qin, K., Wang, J., Li, K. S., et al. (2005). H5N1 virus outbreak in migratory waterfowl. Nature 436, 191-192. doi: 10. 1038/nature 03974

Cheung, C.-L., Rayner, J. M., Smith, G. J. D., Wang, P., Naipospos, T. S. P., Zhang, J., et al. (2006). Distribution of amantadine-resistant H5N1 avian influenza variants in asia. J. Infect. Dis. 193, 1626-1629. doi: 10.1086/504723

Chi, X. S., Bolar, T. V., Zhao, P., Tam, J. S., Rappaport, R., and Cheng, S.-M. (2005). Molecular evolution of human influenza A/H3N2 virus in Asia and Europe from 2001 to 2003. J. Clin. Microbiol. 43, 6130-6132. doi: 10.1128/JCM.43.12. 6130-6132.2005

Cohen, M., Zhang, X. Q., Senaati, H. P., Chen, H. W., Varki, N. M., Schooley, R. T., et al. (2013). Influenza A penetrates host mucus by cleaving sialic acids with neuraminidase. Virol. J. 10:321. doi: 10.1186/1743-422X-10-321

de Jong, R. M. C., Stockhofe-Zurwieden, N., Verheij, E. S., De Boer-Luijtze, E. A., Ruiter, S. J. M., De Leeuw, O. S., et al. (2013). Rapid emergence of a virulent PB2 E627K variant during adaptation of highly pathogenic avian influenza H7N7 virus to mice. Virol. J. 10:276. doi: 10.1186/1743-422X-10-276

Deng, G., Tan, D., Shi, J., Cui, P., Jiang, Y., Liu, L., et al. (2013). Complex reassortment of multiple subtypes of avian influenza viruses in domestic ducks at the Dongting lake region of China. J. Virol. 87:9452. doi: 10.1128/JVI.00 776-13 manuscript. All authors contributed to the article and approved the submitted version.

\section{ACKNOWLEDGMENTS}

We would like to thank all the contributors who isolated and deposited the IAV sequences into the online databases, including GISAID. We extend the thanks to the Research Promotion Division, Department of Research Promotion, Osaka University, Japan, for supporting the English proofreading service of the manuscript.

Dong, G., Peng, C., Luo, J., Wang, C., Han, L., Wu, B., et al. (2015). Adamantane-resistant influenza a viruses in the world (1902-2013): frequency and distribution of M2 gene mutations. PLoS One 10:e0119115. doi: 10.1371/ journal.pone.0119115

Earhart, K. C., Elsayed, N. M., Saad, M. D., Gubareva, L. V., Nayel, A., Deyde, V. M., et al. (2009). Oseltamivir resistance mutation N294S in human influenza A(H5N1) virus in Egypt. J. Infect. Public Health 2, 74-80. doi: 10.1016/j.jiph. 2009.04.004

Eisfeld, A. J., Neumann, G., and Kawaoka, Y. (2015). At the centre: influenza A virus ribonucleoproteins. Nat. Rev. Microbiol. 13, 28-41. doi: 10.1038/ nrmicro3367

El-Shesheny, R., Feeroz, M. M., Krauss, S., Vogel, P., Mckenzie, P., Webby, R. J., et al. (2018). Replication and pathogenic potential of influenza A virus subtypes H3, H7, and H15 from free-range ducks in Bangladesh in mammals. Emerg. Microbes Infect. 7:70. doi: 10.1038/s41426-018-0072-7

Finkelstein, D. B., Mukatira, S., Mehta, P. K., Obenauer, J. C., Su, X., Webster, R. G., et al. (2007). Persistent host markers in pandemic and H5N1 influenza viruses. J. Virol. 81, 10292-10299. doi: 10.1128/JVI.00921-07

Govorkova, E. A., Baranovich, T., Seiler, P., Armstrong, J., Burnham, A., Guan, Y., et al. (2013). Antiviral resistance among highly pathogenic influenza A (H5N1) viruses isolated worldwide in 2002-2012 shows need for continued monitoring. Antivir. Res. 98, 297-304. doi: 10.1016/j.antiviral.2013.02.013

Graef, K. M., Vreede, F. T., Lau, Y.-F., Mccall, A. W., Carr, S. M., Subbarao, K., et al. (2010). The PB2 subunit of the influenza virus RNA polymerase affects virulence by interacting with the mitochondrial antiviral signaling protein and inhibiting expression of beta interferon. J. Virol. 84:8433. doi: 10.1128/JVI. 00879- 10

Heider, H., Adamczyk, B., Presber, H. W., Schroeder, C., Feldblum, R., and Indulen, M. K. (1981). Occurrence of amantadine- and rimantadine-resistant influenza A virus strains during the 1980 epidemic. Acta Virol. 25, 395-400.

Hinshaw, V. S., Bean, W. J., Webster, R. G., and Sriram, G. (1980). Genetic reassortment of influenza A viruses in the intestinal tract of ducks. Virology 102, 412-419. doi: 10.1016/0042-6822(80)90108-7

Hussain, M., Galvin, H. D., Haw, T. Y., Nutsford, A. N., and Husain, M. (2017). Drug resistance in influenza A virus: the epidemiology and management. Infect. Drug Resist. 10, 121-134. doi: 10.2147/IDR.S105473

Jalily, P. H., Duncan, M. C., Fedida, D., Wang, J., and Tietjen, I. (2020). Put a cork in it: plugging the M2 viral ion channel to sink influenza. Antivir. Res. 178:104780. doi: 10.1016/j.antiviral.2020.104780

Järhult, J. D. (2012). Oseltamivir (Tamiflu( $\left({ }^{\circledR}\right)$ ) in the environment, resistance development in influenza A viruses of dabbling ducks and the risk of transmission of an oseltamivir-resistant virus to humans-a review. Infect. Ecol. Epidemiol. 2, doi: 10.3402/iee.v2i0.18385

Joseph, U., Su, Y. C. F., Vijaykrishna, D., and Smith, G. J. D. (2017). The ecology and adaptive evolution of influenza A interspecies transmission. Influenza Other Respir. Viruses 11, 74-84. doi: 10.1111/irv.12412

Kaul, K. L., Mangold, K. A., Du, H., Pesavento, K. M., Nawrocki, J., and Nowak, J. A. (2010). Influenza A subtyping: seasonal H1N1, H3N2, and the appearance of novel H1N1. J. Mol. Diagn. 12, 664-669. doi: 10.2353/jmoldx.2010.09 0225

Kim, J. H., Hatta, M., Watanabe, S., Neumann, G., Watanabe, T., and Kawaoka, Y. (2010). Role of host-specific amino acids in the pathogenicity of avian H5N1 
influenza viruses in mice. J. Gen. Virol. 91, 1284-1289. doi: 10.1099/vir.0. 018143-0

Kim, S. H. (2018). Challenge for one health: co-circulation of zoonotic H5N1 and H9N2 avian influenza viruses in Egypt. Viruses 10:121. doi: 10.3390/ v10030121

Komadina, N., Mcvernon, J., Hall, R., and Leder, K. (2014). A historical perspective of influenza A(H1N2) virus. Emerg. Infect. Dis. 20, 6-12. doi: 10.3201/eid2001. 121848

Lai, S., Qin, Y., Cowling, B. J., Ren, X., Wardrop, N. A., Gilbert, M., et al. (2016). Global epidemiology of avian influenza A H5N1 virus infection in humans, 1997-2015: a systematic review of individual case data. Lancet Infect. Dis. 16, e108-e118. doi: 10.1016/S1473-3099(16)00153-5

Lynch, J. P. III, and Walsh, E. E. (2007). Influenza: evolving strategies in treatment and prevention. Semin Respir. Crit. Care Med. 28, 144-158. doi: 10.1055/s2007-976487

Mao, L., Yang, Y., Qiu, Y., and Yang, Y. (2012). Annual economic impacts of seasonal influenza on US counties: spatial heterogeneity and patterns. Int. J. Health Geogr. 11:16. doi: 10.1186/1476-072X-11-16

Marinova-Petkova, A., Shanmuganatham, K., Feeroz, M. M., Jones-Engel, L., Hasan, M. K., Akhtar, S., et al. (2016). The continuing evolution of H5N1 and H9N2 influenza viruses in Bangladesh between 2013 and 2014. Avian Dis. 60, 108-117. doi: 10.1637/11136-050815-Reg

McCauley, J. W., and Mahy, B. W. (1983). Structure and function of the influenza virus genome. Biochem. J. 211, 281-294. doi: 10.1042/bj2110281

Mehle, A., and Doudna, J. A. (2008). An inhibitory activity in human cells restricts the function of an avian-like influenza virus polymerase. Cell Host Microbe 4, 111-122. doi: 10.1016/j.chom.2008.06.007

Mehle, A., and Doudna, J. A. (2009). Adaptive strategies of the influenza virus polymerase for replication in humans. Proc. Natl. Acad. Sci. U.S.A. 106, 21312 21316. doi: 10.1073/pnas.0911915106

Moasser, E., Moasser, A., and Zaraket, H. (2019). Incidence of antiviral drug resistance markers among human influenza A viruses in the Eastern Mediterranean region, 2005-2016. Infect. Genet. Evol. 67, 60-66. doi: 10.1016/j. meegid.2018.10.023

Molinari, N. A., Ortega-Sanchez, I. R., Messonnier, M. L., Thompson, W. W., Wortley, P. M., Weintraub, E., et al. (2007). The annual impact of seasonal influenza in the US: measuring disease burden and costs. Vaccine 25, 50865096. doi: 10.1016/j.vaccine.2007.03.046

Mostafa, A., Abdelwhab, E. M., Mettenleiter, T. C., and Pleschka, S. (2018). Zoonotic potential of influenza A viruses: a comprehensive overview. Viruses 10:497. doi: 10.3390/v10090497

Musharrafieh, R., Ma, C., and Wang, J. (2020). Discovery of M2 channel blockers targeting the drug-resistant double mutants M2-S31N/L26I and M2S31N/V27A from the influenza A viruses. Eur. J. Pharm. Sci. 141:105124. doi: 10.1016/j.ejps.2019.105124

Nelson, M. I., Simonsen, L., Viboud, C., Miller, M. A., and Holmes, E. C. (2009). The origin and global emergence of adamantane resistant A/H3N2 influenza viruses. Virology 388, 270-278. doi: 10.1016/j.virol.2009.03.026

Nooruzzaman, M., Mumu, T. T., Hasnat, A., Akter, M. N., Rasel, M. S. U., Rahman, M. M., et al. (2019). A new reassortant clade 2.3.2.1a H5N1 highly pathogenic avian influenza virus causing recent outbreaks in ducks, geese, chickens and turkeys in Bangladesh. Transbound. Emerg. Dis. 66, 2120-2133. doi: 10.1111/ tbed.13264

Ormond, L., Liu, P., Matuszewski, S., Renzette, N., Bank, C., Zeldovich, K., et al. (2017). The combined effect of oseltamivir and favipiravir on influenza A virus evolution. Genome Biol. Evol. 9, 1913-1924. doi: 10.1093/gbe/evx138

Orozovic, G., Orozovic, K., Lennerstrand, J., and Olsen, B. (2011). Detection of resistance mutations to antivirals oseltamivir and zanamivir in avian influenza A viruses isolated from wild birds. PLoS One 6:e16028. doi: 10.1371/journal. pone. 0016028

Palekar, R. S., Rolfes, M. A., Arriola, C. S., Acosta, B. O., Guidos, P. A., Vargas, X. B., et al. (2019). Burden of influenza-associated respiratory hospitalizations in the Americas, 2010-2015. PLoS One 14:e0221479. doi: 10.1371/journal.pone. 0221479

Parida, M., Dash, P. K., Kumar, J. S., Joshi, G., Tandel, K., Sharma, S., et al. (2016). Emergence of influenza A (H1N1)pdm09 genogroup 6B and drug resistant virus, India, January to May 2015. Euro Surveill. 21, 6-11. doi: 10.2807/15607917.ES.2016.21.5.30124
Parvin, R., Begum, J. A., Nooruzzaman, M., Chowdhury, E. H., Islam, M. R., and Vahlenkamp, T. W. (2018). Review analysis and impact of co-circulating $\mathrm{H} 5 \mathrm{~N} 1$ and H9N2 avian influenza viruses in Bangladesh. Epidemiol. Infect. 146, 1259-1266. doi: 10.1017/S0950268818001292

Parvin, R., Heenemann, K., Halami, M. Y., Chowdhury, E. H., Islam, M. R., and Vahlenkamp, T. W. (2014). Full-genome analysis of avian influenza virus H9N2 from Bangladesh reveals internal gene reassortments with two distinct highly pathogenic avian influenza viruses. Arch. Virol. 159, 1651-1661. doi: 10.1007/ s00705-014-1976-8

Paules, C. I., and Fauci, A. S. (2019). Influenza vaccines: good, but we can do better. J. Infect. Dis. 219, S1-S4. doi: 10.1093/infdis/jiy633

Principi, N., Camilloni, B., Alunno, A., Polinori, I., Argentiero, A., and Esposito, S. (2019). Drugs for influenza treatment: is there significant news? Front. Med. 6:109. doi: 10.3389/fmed.2019.00109

Proença-Módena, J. L., Macedo, I. S., and Arruda, E. (2007). H5N1 avian influenza virus: an overview. Braz. J. Infect. Dis. 11, 125-133. doi: 10.1590/S141386702007000100027

Rimi, A. N., Hassan, Z. M., Chowdhury, S., Rahman, M., Sultana, R., Biswas, K. P., et al. (2019). A decade of avian influenza in Bangladesh: where are we now? Trop. Med. Infect. Dis. 4:119. doi: 10.3390/tropicalmed4030119

Sharp, G. B., Kawaoka, Y., Jones, D. J., Bean, W. J., Pryor, S. P., Hinshaw, V., et al. (1997). Coinfection of wild ducks by influenza A viruses: distribution patterns and biological significance. J. Virol. 71, 6128-6135. doi: 10.1128/jvi.71.8.61286135.1997

Shin, W. J., and Seong, B. L. (2019). Novel antiviral drug discovery strategies to tackle drug-resistant mutants of influenza virus strains. Expert. Opin. Drug Discov. 14, 153-168. doi: 10.1080/17460441.2019.1560261

Song, W., Wang, P., Mok, B. W.-Y., Lau, S.-Y., Huang, X., Wu, W.-L., et al. (2014). The K526R substitution in viral protein PB2 enhances the effects of E627K on influenza virus replication. Nat. Commun. 5:5509. doi: 10.1038/ncomms6509

Suzuki, Y. (2005). Sialobiology of influenza: molecular mechanism of host range variation of influenza viruses. Biol. Pharm. Bull. 28, 399-408. doi: 10.1248/bpb. 28.399

Takeda, M., Pekosz, A., Shuck, K., Pinto, L. H., and Lamb, R. A. (2002). Influenza a virus M2 ion channel activity is essential for efficient replication in tissue culture. J. Virol. 76, 1391-1399. doi: 10.1128/JVI.76.3.1391-1399.2002

Taubenberger, J. K., and Kash, J. C. (2010). Influenza virus evolution, host adaptation, and pandemic formation. Cell Host Microbe 7, 440-451. doi: 10. 1016/j.chom.2010.05.009

Thomas, J. K., and Noppenberger, J. (2007). Avian influenza: a review. Am. J. Health Syst. Pharm. 64, 149-165. doi: 10.2146/ajhp060181

Tian, J., Qi, W., Li, X., He, J., Jiao, P., Zhang, C., et al. (2012). A single E627K mutation in the $\mathrm{PB} 2$ protein of $\mathrm{H} 9 \mathrm{~N} 2$ avian influenza virus increases virulence by inducing higher glucocorticoids (GCs) level. PLoS One 7:e38233. doi: 10. 1371/journal.pone.0038233

Toots, M., and Plemper, R. K. (2020). Next-generation direct-acting influenza therapeutics. Transl. Res. 220, 33-42. doi: 10.1016/j.trsl.2020.01.005

Trebbien, R., Larsen, L. E., and Viuff, B. M. (2011). Distribution of sialic acid receptors and influenza $A$ virus of avian and swine origin in experimentally infected pigs. Virol. J. 8:434. doi: 10.1186/1743-422X-8-434

Turner, J. C., Feeroz, M. M., Hasan, M. K., Akhtar, S., Walker, D., Seiler, P., et al. (2017). Insight into live bird markets of Bangladesh: an overview of the dynamics of transmission of $\mathrm{H} 5 \mathrm{~N} 1$ and $\mathrm{H} 9 \mathrm{~N} 2$ avian influenza viruses. Emerg. Microbes Infect. 6:e12. doi: 10.1038/emi.2016.142

Vanderlinden, E., and Naesens, L. (2014). Emerging antiviral strategies to interfere with influenza virus entry. Med. Res. Rev. 34, 301-339. doi: 10.1002/med.21289

Wang, P., Song, W., Mok, B. W., Zheng, M., Lau, S. Y., Liu, S., et al. (2019). The PB2 polymerase host adaptation substitutions prime avian indonesia sub clade 2.1 H5N1 viruses for infecting humans. Viruses 11:292. doi: 10.3390/v11030292

Wen, L., Chu, H., Wong, B. H., Wang, D., Li, C., Zhao, X., et al. (2018). Large-scale sequence analysis reveals novel human-adaptive markers in PB2 segment of seasonal influenza A viruses. Emerg. Microbes Infect. 7:47. doi: 10.1038/s41426018-0050-0

WHO (2018). Summary of Neuraminidase Amino Acid Substitutions Associated With Reduced Inhibition by Neuraminidase Inhibitors. Available online at: https://www.who.int/influenza/gisrs_laboratory/antiviral_susceptibility/NAI_ Reduced_Susceptibility_Marker_Table_WHO.pdf?ua=1 (accessed 30 June, 2021). 
Wilcox, B. R., Knutsen, G. A., Berdeen, J., Goekjian, V., Poulson, R., Goyal, S., et al. (2011). Influenza-A viruses in ducks in northwestern minnesota: fine scale spatial and temporal variation in prevalence and subtype diversity. PLoS One 6:e24010. doi: 10.1371/journal.pone.0024010

Wilson, J. C., and von Itzstein, M. (2003). Recent strategies in the search for new anti-influenza therapies. Curr. Drug Targets 4, 389-408. doi: 10.2174/ 1389450033491019

Yamada, S., Hatta, M., Staker, B. L., Watanabe, S., Imai, M., Shinya, K., et al. (2010). Biological and structural characterization of a host-adapting amino acid in influenza virus. PLoS Pathog. 6:e1001034. doi: 10.1371/journal.ppat.1001034

Yin, H., Jiang, N., Shi, W., Chi, X., Liu, S., Chen, J.-L., et al. (2021). Development and effects of influenza antiviral drugs. Molecules 26:810. doi: 10.3390/ molecules 26040810

Yoo, S. J., Kwon, T., and Lyoo, Y. S. (2018). Challenges of influenza A viruses in humans and animals and current animal vaccines as an effective control measure. Clin. Exp. Vaccine Res. 7, 1-15. doi: 10.7774/cevr.2018.7.1.1

Zhang, Y., Aevermann, B. D., Anderson, T. K., Burke, D. F., Dauphin, G., Gu, Z., et al. (2016). Influenza research database: an integrated bioinformatics resource for influenza virus research. Nucleic Acids Res. 45, D466-D474. doi: 10.1093/ nar/gkw857

Zhou, H., Zhang, A., Chen, H., and Jin, M. (2011). Emergence of novel reassortant H3N2 influenza viruses among ducks in China. Arch. Virol. 156, 1045-1048. doi: 10.1007/s00705-011-0940-0
Zürcher, T., Yates, P. J., Daly, J., Sahasrabudhe, A., Walters, M., Dash, L., et al. (2006). Mutations conferring zanamivir resistance in human influenza virus N2 neuraminidases compromise virus fitness and are not stably maintained in vitro. J. Antimicrob. Chemother. 58, 723-732. doi: 10.1093/jac/dk 1321

Conflict of Interest: The authors declare that the research was conducted in the absence of any commercial or financial relationships that could be construed as a potential conflict of interest.

Publisher's Note: All claims expressed in this article are solely those of the authors and do not necessarily represent those of their affiliated organizations, or those of the publisher, the editors and the reviewers. Any product that may be evaluated in this article, or claim that may be made by its manufacturer, is not guaranteed or endorsed by the publisher.

Copyright $(2021$ Hossain, Akter, Dhole, Saha, Kazi, Majbauddin and Islam. This is an open-access article distributed under the terms of the Creative Commons Attribution License (CC BY). The use, distribution or reproduction in other forums is permitted, provided the original author(s) and the copyright owner(s) are credited and that the original publication in this journal is cited, in accordance with accepted academic practice. No use, distribution or reproduction is permitted which does not comply with these terms. 